\title{
Cognitive Side Effects of Cancer Therapy
}

National Cancer Institute

\section{Source}

National Cancer Institute. Cognitive Side Effects of Cancer Therapy. NCI Thesaurus.

Code C115206.

Cog nitive impairment caused by cancer treatment. 